\title{
THE MODE OF IMPULSE CONDUCTION THROUGH THE SPINAL GANGLION
}

\author{
MASAO ITO* AND MASANOBU SAIGA** \\ Department of Physiology, Kumamoto University Medical School
}

It has been known that the spinal ganglion cell of vertebrates, which developed more highly than the shirk, has a monopolar configuration and that its axon ramifies into two branches, i.e., to the dorsal root fiber and to the peripheral nerve fiber, forming a Y-shaped bifurcation (Lenhossék, 1886). An afferent impulse from the peripheral nerve, therefore, conducts through this bifurcation to the dorsal root without passing the cell body (Erlanger, Bishop and Gasser, 1926; Svaetichin, 1951; Dun, 1955). On the other hand in the bipolartyped cell as that found in the spinal ganglion of selachian fishes (Campbell, 1946) afferent impulses travel through the cell body. From the view point of comparative anatomy (Stöhr, 1928) the latter has been believed less favorable to the conduction of afferent impulses than the monopolar-typed cell.

As was reported in the preceding paper (Ito, 1957), the spinal ganglion cell of toads responds to nerve impulses conducted both from the peripheral nerve and from the dorsal root, producing an action potential composed of three component spikes. Two of them, the S and NM spikes, have been confirmed to be initiated from the cell body and from the non-myelinated segment, respectively (Ito, 1959). In this paper it will be shown that the $M$ spike is generated from nodes of Ranvier of the myelinated axon including the one locating at the bifurcation, and the mode of impulse conduction through this bifurcation to the dorsal root fiber, to the peripheral nerve fiber and to the cell body will be compared.

\section{METHODS}

Similar methods to those used in the preceding work (Ito, 1957) were employed to stimulate the spinal ganglion cell of toads (Bufo vulgaris formosus) and to record potentials intracellularly from it. In order to study the impulse conduction through the ganglion, two pairs of silver wire electrodes, each of which was used for stimulation or recording alternatively, were set on the peripheral nerve and the dorsal root at a distance of about $2 \mathrm{~cm}$. from the ganglion. $1 \%$ and $2 \%$ urethane Ringer solutions, in which the ganglion was immersed, were used for testing the safety factor of the conduction. The room temperature was between 10 and $20^{\circ} \mathrm{C}$.

\footnotetext{
Received for publication July 14, 1958.

* 伊藤正男

Present address: Department of Physiology, Faculty of Medicine, University of Tokyo. ** 斎賀正信
} 


\section{1. $M$ spike under the hyperpolarisation}

When an action potential was evoked in the spinal ganglion cell by a stimulus delivered to the peripheral nerve, its rising phase showed two inflections indicating three-step conduction from the $\mathrm{M}$ to the $\mathrm{S}$ spike (Ito, 1957). The same inflections were also observed on the action potential evoked by stimulating the dorsal root (marked by dots and arrows in fig. 1). Therefore, the sites generating these three component spikes, the S, NM and M spikes, should be common to both paths from the peripheral nerve and from the dorsal root.

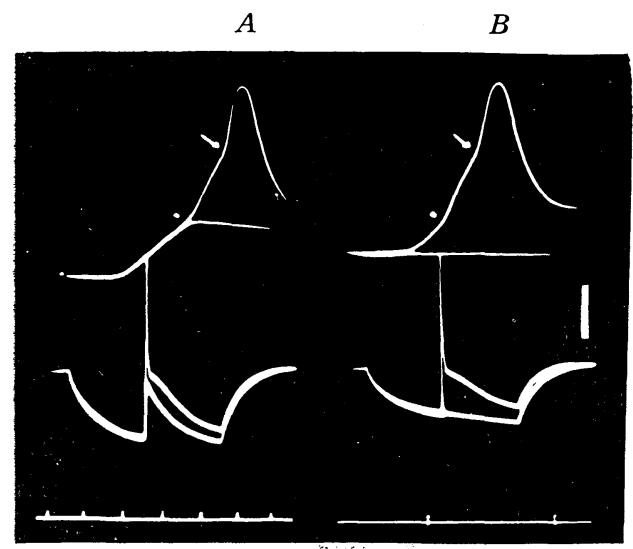

FIG. 1. The conduction block by the hyperpolarisation. The cell membrane was hyperpolarised by applying rectangular currents through the intracellular electrode, and either the peripheral nerve $(A)$ or the dorsal root $(B)$ was stimulated about $50 \mathrm{msec}$. after the onset of the hyperpolarising current (lower records). Each record was obtained by superimposing two successive sweeps in order to show the critical condition for block. The action potential evoked during the hyperpolarisation is seen in upper traces, which were obtained by the second oscilloscope triggered by stimulating pulses. Time markers; 1 msec. for the upper traces (left) and $100 \mathrm{msec}$. for the lower ones (right). Voltage calibration; $50 \mathrm{mV}$ for the upper traces. $10^{\circ} \mathrm{C}$.

When the cell membrane was hyperpolarised by currents applied through the intracellular electrode, the impulse conducted from the peripheral nerve was usually blocked between the $\mathrm{M}$ and NM spikes (fig. $1(A)$ ), but the $\mathrm{M}$ spike was hardly abolished even by the hyperpolarisation larger than $100 \mathrm{mV}$. On the other hand, when the spikes were induced by the stimulation of the dorsal root, the block of the $\mathrm{M}$ spike was observed (fig. $1(B)$ ). The magnitude of the hyperpolarisation required for this block was usually smaller than that necessary for the M-NM block in the same cell (table 1). This fact that different hyperpolarisations were required for blocking the same $M$ spike points out that the nerve impulses initiated from the peripheral nerve and that from the dorsal root travel to the site generating the $M$ spike through different paths from each other. Consequently, the site, where the $M$ spike is generated, should include the node of Ranvier locating at the bifurcation of the myelinated axon. The pre-M spike, whereby the M spike is initiated, should be originated from the node of Ranvier in the peripheral nerve or in the dorsal root locating close to the bifurcation. The central pre-M spike, generated from the dorsal root, was usually smaller than $1 \mathrm{mV}$, when observed under the hyperpolarisation (fig. $1(B))$. The peripheral pre-M spike was hardly isolated in the present experiments. Moreover, even when one or two potentials were separated from the M spike (fig. 2), it was usually impossible to decide whether these poten- 
tials should be attributed to the pre-M spike or to components of the $\mathrm{M}$ spike, because the action potential conducted from the dorsal root was blocked by a too small hyperpolarisation to reveal that the same potentials were included in its configuration. The axon of the spinal ganglion cell has been known to

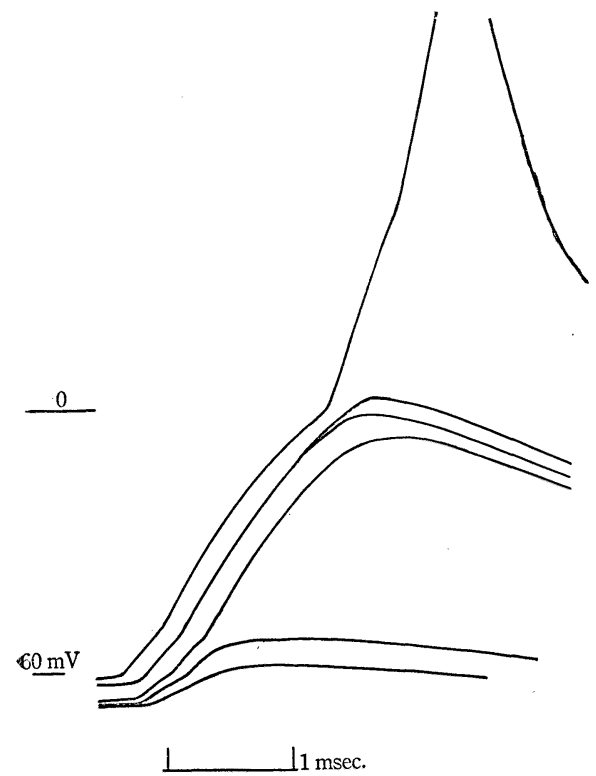
have one or two nodes between the bifurcation and the non-myelinated segment (Lenhossék, 1886). It is possible that the conduction between these nodes was blocked by the hyperpolarisation. The name of the $M$ spike, therefore, should be applied to the compound spike potentials originated from nodes of Ranvier, situated between the bifurcation and the non-myelinated segment and including the one at the bifurcation.

FIG. 2. Traces of a photograph showing a large $\mathrm{M}$ spike under the hyperpolarisation. Two small steps appeared on the foot of the M spike, from which small spike potentials were separated by a hyperpolarisation larger than $60 \mathrm{mV}$. The line $(0)$ shows the original resting level. Stimuli were applied to the peripheral nerve.

TABLE 1

\begin{tabular}{c|c|c}
\hline \multirow{2}{*}{ Cell No. } & \multicolumn{2}{|c}{ Hyperpolarisation required for } \\
\cline { 2 - 3 } & $\begin{array}{c}\text { M-NM block } \\
(\mathrm{mV})\end{array}$ & $\begin{array}{c}\text { Central pre-M-M block } \\
(\mathrm{mV})\end{array}$ \\
\hline 1 & 25 & 15 \\
2 & 37 & 8 \\
3 & 45 & 26 \\
4 & 57 & 44 \\
5 & 61 & 21 \\
\hline Average of 16 cells & 45 & \\
\hline
\end{tabular}

\section{Refractory period}

When two successive stimuli were applied to the peripheral nerve or to the dorsal root, the response of the cell to the second stimulus suffered from changes in its configuration as was observed in motoneurones (Brock, Coombs and Eccles, 1953). By stimulating the peripheral nerve, when the time-interval of the successive spike initiation was reduced to $2-6 \mathrm{msec}$., the conduction from the $\mathrm{M}$ to the $\mathrm{S}$ spike was affected. It was commonly observed that the $\mathrm{M}$ spike remained isolated during this refractory period, and the isolated NM spike was rarely observed (fig. $3 A, a$ ). The least interval of the M-NM conduction was 
longer than that of the NM-S conduction in almost all cells examined. Further shortening of the interval caused a decrease of the $M$ spike magnitude, and at the peak of the after-positivity following the spike potential evoked by the conditioning shock the M spike usually failed to be induced. However, as the membrane resistance of the cell body was reduced at the peak of the afterpositivity (Ito and Saiga, unpublished), it is possible that the $M$ spike could not be recorded although it might have been initiated. Therefore, the least interval of the conduction from the peripheral pre- $M$ to the $M$ spike was not measured accurately in this experiment. On the other hand the spike potential produced by stimulating the dorsal root abruptly broke down to a minute potential, when the interval of the spike initiation was shortened to 4-8 msec. (fig. $3 B, a$ ), without a stage where the $\mathrm{M}$ or the NM spike was isolated. This fact shows that the conduction from the central pre-M to the $M$ spike was blocked before the M-NM or the NM-S block occurred. The least interval of the former is shown in table 2, where that of the M-NM conduction measured by stimulating the peripheral nerve in the same cell is also listed for compari-

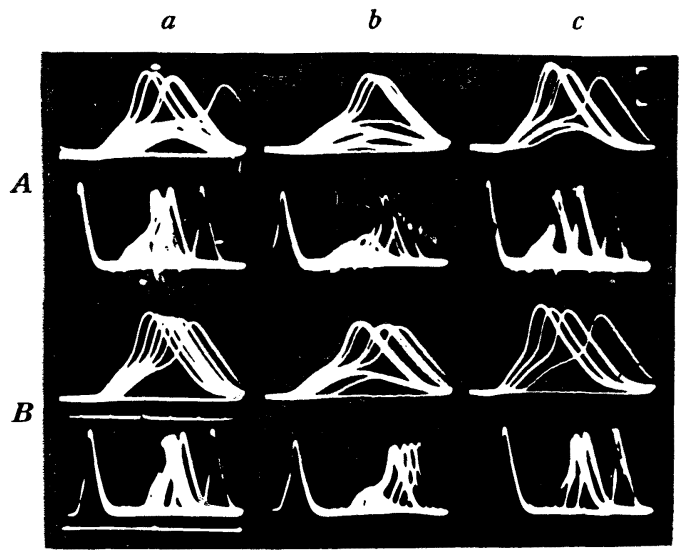

FIG. 3. Responses of the spinal ganglion cell during the refractory period. $A$; responses evoked by stimulating the peripheral nerve. $B$; those obtained by stimulating the dorsal root. Records in column $a$ were obtained without polarisation and those in $b$ and $c$ under the depolarisation of $9 \mathrm{mV}$ and under the hyperpolarisation of $18 \mathrm{mV}$, respectively. In this experiment the cell was polarised by steady currents and the photographs were taken about 3 minutes after the onset of the polarisation. 7-10 sweeps, repeating once per second, were superposed by changing the stimulus interval successively. The upper traces, obtained by the second oscilloscope which was triggered by the test stimuli, show the action potential in the refractory period with a faster sweep velocity. Time markers; $1 \mathrm{msec}$. for the upper traces and $10 \mathrm{msec}$. for the lower ones. Voltage calibration; $50 \mathrm{mV}$ for the upper traces. $10^{\circ} \mathrm{C}$.

TABLE 2

\begin{tabular}{|c|c|c|}
\hline \multirow{2}{*}{ Cell No. } & \multicolumn{2}{|c|}{ Least interval of the conduction between } \\
\hline & $\begin{array}{l}M \text { and NM spikes } \\
\text { (msec.) }\end{array}$ & $\begin{array}{l}\text { Central per-M and } M \\
\text { spikes (msec.) }\end{array}$ \\
\hline $\begin{array}{l}1 \\
2 \\
3 \\
4 \\
5\end{array}$ & $\begin{array}{l}2.5 \\
4.0 \\
2.7 \\
3.8 \\
5.6\end{array}$ & $\begin{array}{l}4.8 \\
4.2 \\
6.6 \\
6.7 \\
6.9\end{array}$ \\
\hline Average of 8 cells & 3.9 & 5.9 \\
\hline
\end{tabular}

Measrements were done at $10-16^{\circ} \mathrm{C}$. 
son. It has been observed in section 1 that a smaller hyperpolarisation was necessary for blocking the conduction from the central pre- $M$ to the $M$ spike than that from the peripheral pre-M spike. These facts point out that the former has a smaller safety factor than the latter.

When the cell membrane was depolarised by currents applied through the impaled microelectrode, the least interval of the M-NM or the NM-S conduction became longer (fig. $3 b$ ), and the isolated $\mathrm{M}$ or NM spike was obtained even by stimulating the dorsal root. In contrast to this the least interval of the conduction from the pre- $\mathrm{M}$ to the $\mathrm{M}$ spike was not altered significantly either by the depolarisation or by the hyperpolarisation (fig. $3 c$ ).

Fuortes, Frank and Becker (1957) showed that by the transmembrane stimulation of the spinal motoneurones the IS spike, separated from the SD spike, was evoked during the refractory period. This may supply an evidence indicating that the inflected rising phase of the action potential produced by the transmembrane stimulation is formed by the superposition of the SD spike on the IS spike ( $B$ potential on $A$ potential by their notation). In spinal ganglion cells, the NM spike was hardly separated from the $S$ spike during the refractory period, but similar observations to Fuortes $e t$ al. were made in rather rare cases.

\section{Repetitive activation of the spinal ganglion cell}

Fig. 4 shows an example of the response of the ganglion cell repetitively activated at $10^{\circ} \mathrm{C}$. With the stimulus frequency of $10-30 \mathrm{c} / \mathrm{sec}$., the action potential became smaller in its magnitude and slower in its time course, and its after-positivity was lost ( $c f$. Frankenhaeuser and Hodgkin, 1956) (fig. $4 A, B$ ). An increase of the frequency caused the inflection of its rising phase more prominent, and with the frequency of $50-60 \mathrm{c} / \mathrm{sec}$. the intermittent conduction block occurred between the NM and S spikes $(C)$ and with that of $80-100 \mathrm{c} / \mathrm{sec}$. between the $\mathrm{M}$ and NM spikes $(D)$. When the stimulus frequency was increased further, the NM-S block occurred continuously (fig. $5 A$ and $B$, with $100 \mathrm{c} / \mathrm{sec}$.) and the block between the pre-M and the $M$ spikes was also observed ( $C$ and $D$, with $130 \mathrm{c} / \mathrm{sec}$.).

The effect of the repetitive activation was dependent on the temperature. The relation between the temperature and the minimum frequency necessary for blocking the conduction is shown in table 3 for the various types of block. It shows that the conduction from the NM to the S spike was blocked with a frequency lower than $200 \mathrm{c} / \mathrm{sec}$. even at $20^{\circ} \mathrm{C}$., whereas the $\mathrm{M}$ spike followed each stimulus with a frequency as high as $800 \mathrm{c} / \mathrm{sec}$. In the repetitive activation the NM-S conduction was blocked at lower frequency than the M-NM conduction, while the least interval measured by two successive stimuli was longer in the latter than in the former (section 2). These paradoxical facts suggest that in the course of the repetitive activation the refractory period of the cell body was more rapidly prolonged than that of the non-myelinated segment. However, a prolongation of the refractory period of the latter was also observed. The M-NM conduction was blocked by a stimulus interval longer than the least interval measured by two successive stimuli ( $c f$. Gasser and Grundfest, 1936). 


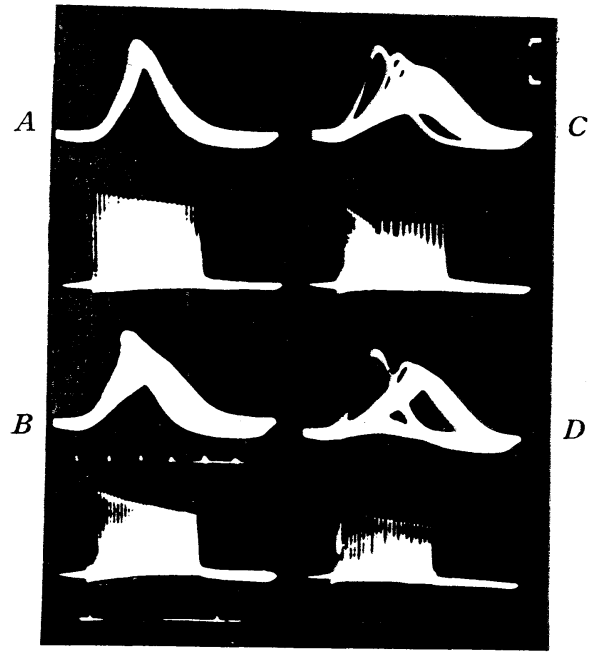

FIG. 4. Repetitive activation of the spinal ganglion cell. Stimulus frequency; $26 \mathrm{c} / \mathrm{sec}$. in $A, 36 \mathrm{c} / \mathrm{sec}$. in $B, 60 \mathrm{c} / \mathrm{sec}$. in $C$ and $80 \mathrm{c} / \mathrm{sec}$. in $D$. The conduction was blocked intermittently in $C$ between the NM and S spikes, and in $D$ between the $\mathrm{M}$ and NM spikes. Upper traces in each record show the action potential with a faster sweep velocity, being obtained by the second oscilloscope, the timebase of which was triggered once by three stimulating pulses. Time markers; $1 \mathrm{msec}$. for the upper traces and $1 \mathrm{sec}$. for the lower ones. Voltage calibration; $50 \mathrm{mV}$ for the upper traces. $10^{\circ} \mathrm{C}$.

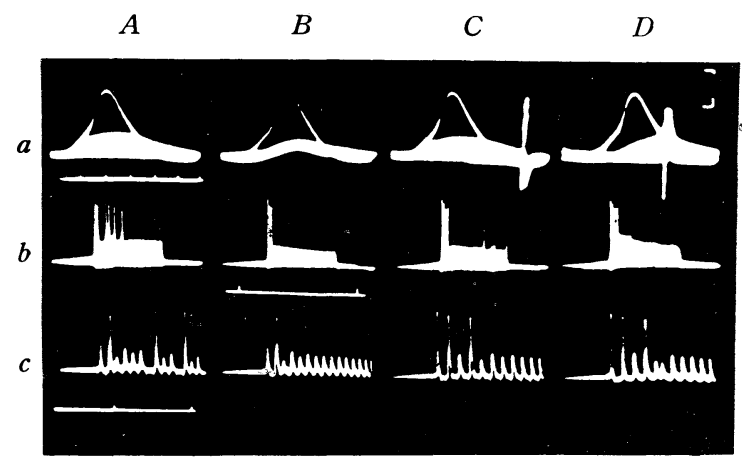

FIG. 5. Continuous conduction block caused by a high frequency stimulation. Responses of the cell are shown in two different sweep velocities in $b$ and $c$, being obtained by the first oscilloscope, and in a the action potentials are recorded by the second oscilloscope triggered by the stimulating pulses. $A$ and $C$; dorsal root stimulation. $B$ and $D$; peripheral nerve stimulation. Stimulus frequency; 100 $\mathrm{c} / \mathrm{sec}$. in $A$ and $B, 130 \mathrm{c} / \mathrm{sec}$. in $C$ and $D$. Time markers; 1 msec. for $a, 100 \mathrm{msec}$. for $c$ and $1 \mathrm{sec}$. for $b$. Voltage calibration; $50 \mathrm{mV}$ for the uppermost records. $10^{\circ} \mathrm{C}$.

TABLE 3. The minimum frequency of the repetitive activation necessary for blocking the conduction between the NM and S spikes $\left(f_{s}\right)$, between the $\mathrm{M}$ and NM spikes $\left(f_{n m}\right)$ and to the $\mathrm{M}$ spike $\left(f_{m}\right)$ at various temperatures. Repetitive stimuli lasting about $1 \mathrm{sec}$. were applied to the peripheral nerve at a distance of about $2 \mathrm{~cm}$. from the ganglion

\begin{tabular}{c|c|c|c|c}
\hline Cell No. & $\begin{array}{c}\text { Temperature } \\
\left({ }^{\circ} \mathrm{C} \text {. }\right)\end{array}$ & $\begin{array}{c}f_{s} \\
\text { (c/sec. })\end{array}$ & $\begin{array}{c}f_{n m} \\
\text { (c/sec. })\end{array}$ & $\begin{array}{c}f_{m} \\
\text { (c/sec. })\end{array}$ \\
\hline 1 & 10 & 60 & 80 & 110 \\
2 & 16 & 120 & 220 & 300 \\
3 & 18 & 200 & 240 & 500 \\
4 & 20 & 140 & 300 & 800 \\
\hline
\end{tabular}


The presence of the $M$ spike in the intracellular record shows that the impulse from the peripheral nerve arrives at the bifurcation, but it does not immediately indicate that the same impulse further conducts to the dorsal root. However, Svaetichin (1951) observed that the impulse, elicited by stimulating a single fiber in the peripheral nerve, conducted to the dorsal root even when the block was noticed in the extracellular potential of the ganglion cell. In the present experiments it was also observed that the spike potential recorded from the whole dorsal root did not show any appreciable decrease with stimulus frequencies where the M-NM or the NM-S block occurred. With these frequencies a train of impulses might have traversed the ganglion without block.

\section{Effects of urethane on the impulse conduction through the ganglion}

It has been shown in section 1 and 2 that the conduction of the impulse from the dorsal root to the bifurcation has a smaller safety factor than that from the peripheral nerve. The narcotics affected also the centrifugal conduction through the ganglion much more than the centripetal one. As is shown in fig. 6, when the ganglion was immersed in the Ringer solution containing $1 \%$ urethane, the compound spike potential of the peripheral nerve, caused by the dorsal root stimulation, was almost completely abolished, while that of the dorsal root obtained by stimulating the peripheral nerve was less affected. It was observed intracellularly that under a 1\% urethane Ringer solution the dorsal root stimulation failed to evoke the $M$ spike, while the peripheral nerve stimu-

$A$
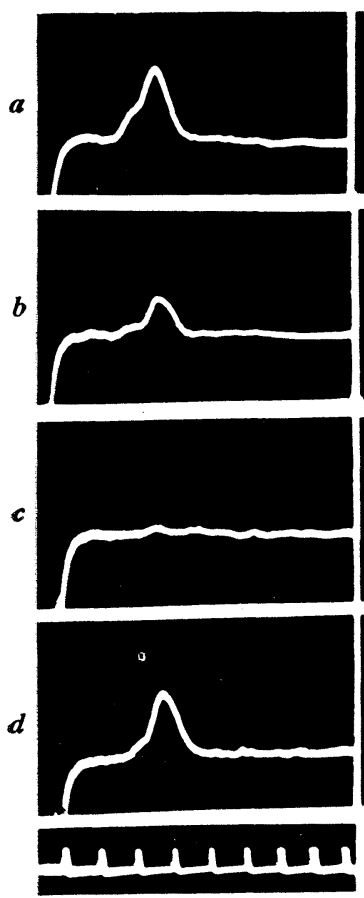

$B$
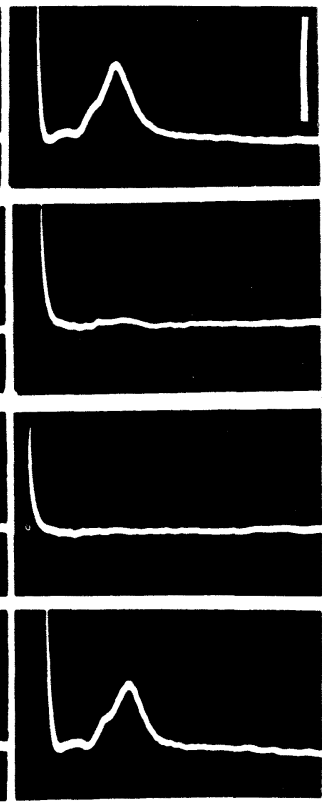
lation set up the one isolated from the NM-S complex. The magnitude of the $M$ spike was reduced gradually in this solution, but it was not abolished in almost all cells. Therefore, fig. $6 b$ may represent a stage where the centrifugal conduction was blocked between the dorsal root and the bifurcation,

FIG. 6. Effects of urethane on the impulse conduction through the spinal ganglion. $\boldsymbol{A}$; spike potential recorded from the dorsal root, which was in. duced with a supramaximal stimulus applied to the peripheral nerve. $B$; that of the peripheral nerve obtained by stimulating the dorsal root. $a$; before the test solution was applied. $b$; $25 \mathrm{~min}$. after the ganglion was immersed in a $1 \%$ urethane Ringer solution. $c ; 2$ min. after the $2 \%$ urethane solution was applied. $d$; $10 \mathrm{~min}$. after the ganglion was recovered in the normal Ringer solution. Voltage calibration; $1 \mathrm{mV}$. Time marker; $1 \mathrm{msec}$. $20^{\circ} \mathrm{C}$. 
but the centripetal one was maintained. The reduction of the spike amplitude recorded from the dorsal root (fig. $6 b, A$ ) should indicate that the centripetal conduction from the bifurcation to the dorsal root was blocked partly. Consequently, the safety factor of the conduction from the bifurcation to the dorsal root might be smaller than that from the peripheral nerve to the bifurcation, as was argued by Dun (1955), but it should be larger than that from the dorsal root to the bifurcation. Under a $2 \%$ urethane solution both the centrifugal and centripetal conductions were nearly completely blocked (fig. 6c). Corresponding to this, the $\mathrm{M}$ spike was not observed in the intracellular record by stimulating the dorsal root as well as the peripheral nerve.

\section{DISCUSSION}

It has been shown in the present experiments that the nerve impulse travels to the bifurcation from the dorsal root with a smaller safety factor than from the peripheral nerve. This fact may be explained by following two reasons. First, the diameter of the dorsal root fiber is two third as small as that of the peripheral nerve fiber (Ito and Oshima, unpublished), by which it can be expected that the safety factor of the conduction between the bifurcation and the dorsal root, either centripetal or centrifugal, should be lowered than that along the peripheral nerve fiber because of the larger longitudinal resistance of the axoplasm in the former than in the latter. Second, Lenhossék (1886) observed on frogs that the unmyelinated membrane at the bifurcation had a considerably larger area than an ordinary nodal membrane. If this holds true for toads' spinal ganglion, the safety factor of the conduction to the bifurcation either from the peripheral nerve or from the dorsal root would have a smaller safety factor than that in the inverse direction, because in the former case the action current generated from a smaller area of an ordinary nodal membrane should stimulate the bifurcation having a larger area. The centrifugal conduction between the dorsal root and the bifurcation should be affected by both factors, while that along the inverse direction or the centripetal one from the peripheral nerve to the bifurcation should be influenced by one of them. There might remain other reasons which should be taken into consideration, and a further investigation on this problem will be dealt with in the succeeding report.

The repetitive activation affected the conduction from the bifurcation to the cell body more than that through the bifurcation from the peripheral nerve to the dorsal root. Therefore, if the spinal ganglion cell has a bipolar type, the successive conduction of the sensory impulses would be blocked when they pass through the cell body. This agrees with the view of the comparative anatomy that the monopolar-typed cell of the spinal ganglion cell is more favorable to the sensory conduction than the bipolar-typed one.

Steinach (1899) showed that even when the spinal ganglion was deprived of the blood supply and its cells showed a sign of degeneration, the reflex activity through this ganglion was not affected. From this observation he concluded that the centripetal conduction was characterized by its complete independence of the ganglion cell. This has been confirmed in the present experiments because under normal conditions the cell responded always only once 
to one impulse either centripetal or centrifugal (Ito, 1957) and because any functions of the cell to modify the impulse pattern passing through the ganglion were not detected. Therefore, although the cell responds to both the nerve impulse from its axon and the direct stimulus applied to its membrane in the same way as spinal motoneurones or other nerve cells do, it should be mentioned here that this activity of the spinal ganglion cell has no active role in the conduction of the afferent impulses.

\section{SUMMARY}

1. The conduction of the impulse through toad spinal ganglion was studied by analysing the action potential obtained from the ganglion cells with an intracellular electrode and by recording the conducted spike potential from the peripheral nerve or from the dorsal root.

2. By making the cell hyperpolarised and refractory the site generating the M spike, the smallest component of the intracellular spikes, was confirmed to be nodes of Ranvier locating between the non-myelinated segment and the bifurcation and including the one at the bifurcation.

3. The conduction from the dorsal root to the bifurcation was blocked by a smaller hyperpolarisation than that from the peripheral nerve, and the least interval of the former was longer than that of the latter.

4. Urethane affected the centrifugal conduction through the spinal ganglion much more than the centripetal one, indicating that the conduction from the bifurcation to the dorsal root has a larger safety factor than that of the inverse direction.

5. Repetitive activation of the nerve affected the conduction from the bifurcation to the cell body more than that through the bifurcation from the peripheral nerve to the dorsal root. This was correlated with the development of the ganglion cell from a bipolar type in fishes to a monopolar one in higher vertebrates.

Grateful acknowledgement is made to Dr. Masayasu Sato for his encouragement and criticism during this investigation and manuscription. The authors are also indebted to Dr. Susumu Hagiwara for his kind advices during the manuscription.

\section{REFERENCES}

1. BROCK, L. G., COOMBS, J. S. AND ECCLES, J. C. Intracellular recording from antidromically activated motoneurones. J. Physiol. 122: 429-461, 1953.

2. CAMPBELL, B. Morphology and Conduction of bipolar dorsal root ganglion cells of selachian fishes. J. Neurophysiol. 9 : 173-179, 1946.

3. Dus, F. T. The delay and blockage of sensory impulses in the dorsal root ganglion. J. Physiol. 127 : 252-264, 1955.

4. ERlanger, J., Bishop, G. AND GASSER, H. S. The action potential waves transmitted between the sciatic nerve and its spinal dorsal root. Amer. J. Physiol. 78: 574-591, 1926.

5. Frankenhaeuser, B. AND Hodgkin, A. L. The after-effects of impulses in the giant nerve fibres of Loligo. J. Physiol. 131: 341-376, 1956. 
6. Furotes, M. G. F., Frank, K. AND BeCKer, MARy C. Steps in the production of motoneuron spikes. J. gen. Physiol. 40: 735-752, 1957.

7. Gasser, H. S. AND GRUNDFEST, H. Action and excitability in mammalian A fibres. Amer. J. Physiol. 117: 113-133, 1936.

8. ITO, M. The electrical activity of spinal ganglion cells investigated with intracellular microelectrodes. Jap. J. Physiol. 7: 297-323, 1957.

9. ITO, M. An analysis of potentials recorded intracellularly from the spinal ganglion cell. Jap. J. Physiol. 9 : in press, 1959.

10. Lenhossék, M. v. Untersuchungen über die Spinalganglien des Frosches. Arch. mikr. Anat. $26: 370-453,1886$.

11. Steinach, E. Ueber die centripetale Erregungsleitung im Bereiche des Spinalganglions. Pfiuger's Arch. ges. Physiol. 78 : 291-414, 1899.

12. STÖHR, P. Das peripherische Nervensystems. pp. 143-201 in von Möllendorf's Handbuch der mikroskopischen Anatomie des Menschen, Bd. 4. Berlin: Julius Springer, 1928.

13. Svaetichin, G. Analysis of action potentials recorded from single spinal ganglion cells. Acta Physiol. Scandinav. 24 : Suppl. 86, 23-57, 1951. 